\title{
Lymphadenoma of the salivary gland: clinicopathological and immunohistochemical analysis of 33 tumors
}

Raja R Seethala ${ }^{1}$, Lester DR Thompson ${ }^{2}$, Douglas R Gnepp ${ }^{3}$, E Leon Barnes ${ }^{1}$, Alena Skalova ${ }^{4}$, Kathleen Montone ${ }^{5}$, Shubhada Kane ${ }^{6}$, James S Lewis $\mathrm{Jr}^{7}$, Lynn W Solomon ${ }^{8}$, Roderick HW Simpson ${ }^{9}$, Ashraf Khan ${ }^{10}$ and Manju L Prasad ${ }^{11}$

${ }^{1}$ Departments of Pathology, Presbyterian University Hospital, Pittsburgh, PA, USA; ${ }^{2}$ Woodland Hills Medical Center, Woodland Hills, CA, USA; ${ }^{3}$ Rhode Island Hospital of Brown University, Providence, RI, USA;

${ }^{4}$ Medical Faculty of Charles University, Prague, Czech Republic; ${ }^{5}$ Hospital of the University of Pennsylvania, Philadelphia, PA, USA; ${ }^{6}$ Tata Memorial Hospital, Mumbai, India; ${ }^{7}$ Washington University School of Medicine, St Louis, MO, USA; ${ }^{7}$ Tufts University School of Dental Medicine, Boston, MA, USA; ${ }^{9}$ Royal Devon and Exeter Hospital, Exeter, UK; ${ }^{10}$ UMass Memorial Medical Center, Worcester, MA, USA and ${ }^{11}$ Yale School of Medicine, New Haven, CT, USA

Lymphadenomas (LADs) are rare salivary gland tumors. Their clinicopathologic characteristics and etiopathogenesis are poorly understood. We examined 33 LADs in 31 patients (17 women and 14 men) aged 11-79 years (median 65 years). There were 22 sebaceous LADs in 21 patients ( 9 women and 12 men) and 11 nonsebaceous LADs in 10 patients (8 women and 2 men). Two patients had synchronous double tumors. Twentysix tumors $(79 \%)$ arose in parotid, three in the neck, and two each in submandibular gland and oral cavity. Extraparotid tumors were seen in 2 of $21(10 \%)$ patients with sebaceous and 4 of $10(40 \%)$ patients with nonsebaceous LADs. Seven of twenty-three (30\%) patients had immunosuppressive therapy for unrelated diseases. The tumors were well circumscribed, encapsulated $(n=28,84 \%)$ painless masses, varying in size from 0.6 to $6 \mathrm{~cm}$ (median 2.2). The cut surfaces were gray-tan to yellow, homogeneous and multicystic $(n=24,72 \%)$. The epithelial cells were basaloid, squamous and glandular, forming solid nests, cords, tubules, and cysts. Sebaceous differentiation was restricted to sebaceous lymphadenoma. The epithelial cells expressed basal cell markers (p63, 34BE12, and/or CK5/6, 18/18, 100\%) and the luminal glandular cells expressed CK7 (12/12, 100\%). Myoepithelial cells were absent $(n=10 / 16,63 \%)$ or focal. The lymphoid stroma was reactive, with germinal centers in 28 (84\%). There was no evidence of HPV (0/11), EBV (0/7), and HHV-8 (0/8). Malignant transformation to sebaceous and basal cell adenocarcinoma was seen in one patient each. None of the 11 patients with followup (1-8 years) recurred. In summary, sebaceous and non-sebaceous LADs are benign, encapsulated, solid and cystic tumors affecting older adults. Non-sebaceous LADs affect women and extraparotid sites more frequently than sebaceous LADs. Altered immune status may have a role in their etiopathogenesis. Multiple synchronous tumors, origin in buccal mucosa, and malignant transformation may rarely occur.

Modern Pathology (2012) 25, 26-35; doi:10.1038/modpathol.2011.135; published online 2 September 2011

Keywords: benign; lymphadenoma; neoplasm; salivary gland

Correspondence: Associate Professor ML Prasad, MD, Department of Pathology, Yale University School of Medicine, PO Box 208070 , 310 Cedar Street, New Haven, CT 06520, USA.

E-mail: manju.prasad@yale.edu

Received 17 February 2011; revised 8 June 2011; accepted 8 June 2011; published online 2 September 2011
Lymphadenomas (LADs) are rare benign tumors of the major salivary glands comprising biphasic tumor cell population that show groups of epithelial cells with or without sebaceous differentiation admixed with intense, reactive lymphoid proliferation. Since its first description in 1960 by McGavran et al, ${ }^{1}<75$ cases $^{2-5}$ have been published, the largest series comprising three cases only. ${ }^{6}$ The clinicopathological 
features are poorly understood, and the etiology is unknown. LADs are typically tumors of the adults, and present as painless mass of long duration in the parotid gland. In most tumors, the epithelial component comprising benign basal and squamous cells with sebaceous differentiation. A much smaller number of tumors lack sebaceous differentiation. ${ }^{7}$ Only 11 non-sebaceous LAD are reported in the literature. ${ }^{5,6,8-16}$ We present a multicenter experience with 33 salivary LAD: 22 sebaceous and 11 non-sebaceous, in order to better define their clinicopathological characteristics, and explore their etiopathogenesis including association with malignancy, altered immune status, and viral infection.

\section{Materials and methods}

The study comprising $33 \mathrm{LAD}$ diagnosed at 10 different institutions by head and neck pathologists with several years of experience in salivary gland pathology. Clinical information, pathologic findings, and results of immunohistochemistry (IHC) were analyzed (Table 1). In order to explore viral etiology, strong and diffuse $(>80 \%)$ cytoplasmic and nuclear expression of p16 was considered a surrogate marker of high risk type HPV infection, as described in tonsillar squamous cell carcinoma. In situ hybridization for HPV was performed in tumors strongly and diffusely positive for $\mathrm{p} 16^{17}$ using a wide spectrum HPV-DNA probe set (code Y1404, Dako, Carpinteria, CA), followed by subtyping, if positive. In situ hybridization for EBV-encoded RNA (EBER) was performed. One of the patients was included in a series of sclerosing polycystic adenosis, ${ }^{18}$ and another was the subject of a case report on bilateral intraoral sebaceous LAD. ${ }^{19}$

\section{Results}

Twenty-two sebaceous and eleven non-sebaceous LADs were identified in thirty-one patients (Tables
$2-4)$. The tumors were solitary in 29 patients (94\%). A 54-year-old man developed bilateral sebaceous LAD as pedunculated masses in the right and left posterior buccal mucosa (patient SL18a,b in Table 3), and a 70-year-old man suffering from chronic lymphocytic leukemia developed two non-sebaceous LAD in right intraparotid lymph nodes (patient NSL8a,b in Table 4).

\section{Clinical Features}

Table 2 summarizes the age and sex distribution, and the tumor size, and Tables 3 and 4 show clinical features in the two subtypes of LAD. All but five patients were older than 50 years (84\%), and only one was a child: an 11-year-old girl with nonsebaceous LAD (patient NSL7; Table 4). Twenty-six tumors $(79 \%)$ arose in the parotid, two in the submandibular salivary gland, and two in the buccal mucosa. One tumor arose within the right cervical lymph node and in two tumors the location was reported as 'left neck'. Nineteen of twenty-one $(90 \%)$ patients had intraparotid sebaceous LAD, compared with 6 of $10(60 \%)$ patients with nonsebaceous LAD. Thirty tumors presented as painless masses, some slowly enlarging (Figure 1a). The duration of symptoms, when known, ranged from a few months to several years.

Medical history was available in 23 patients, and 11 of them (48\%) had co-morbidities that included diabetes, hypertension, hyperlipidemia, coronary artery disease, depression, hypothyroidism, obesity, autoimmune and neoplastic diseases. Five patients had malignancies: basal cell carcinoma and squamous cell carcinoma of the skin in one patient each (patients SL7 and SL16, respectively; Table 3), breast carcinoma in one (patient SL20; Table 3), low-grade salivary duct carcinoma in one (patient NSL4; Table 4), and chronic lymphocytic leukemia in one patient (patient NSL8; Table 4). Two patients were on immunomodulatory drugs for rheumatoid arthritis

Table 1 Immunohistochemistry panel

\begin{tabular}{|c|c|c|c|c|}
\hline Antibody & Clone & Dilution & Antigen retrieval & Vendor \\
\hline Calponin & CALP & $1: 500$ & Dako TRS & Dako, Carpinteria, CA \\
\hline CD3 & Rabbit polyclonal & $1: 200$ & Dako TRS & Dako \\
\hline CD5 & $4 \mathrm{C} 7$ & $1: 100$ & Dako TRS & Vector, Burlingame, CA \\
\hline CD20 & L26 & $1: 1000$ & Dako TRS & Dako \\
\hline CD79a & HM57 & $1: 50$ & Dako TRS & Dako \\
\hline CK5/6 & $\mathrm{D} 5 / 16 \mathrm{~B} 4$ & $1: 50$ & EDTA pH8 & Dako \\
\hline CK7 & OV-TL 12/30 & $1: 200$ & Dako TRS & Dako \\
\hline CK20 & $\mathrm{K}_{\mathrm{S}} 20.8$ & $1: 200$ & Dako TRS & Dako \\
\hline EBV-LMP & CS 1-4 & $1: 50$ & Trypsin & Dako \\
\hline HHV-8 & 13B10 & $1: 25$ & Dako TRS & Novocastra, UK \\
\hline HMWK & $34 \beta \mathrm{E} 12$ & $1: 50$ & Dako TRS & Dako \\
\hline Ki-67 & MIB-1 & $1: 200$ & Dako TRS & Dako \\
\hline p16 & $\mathrm{E} 6 \mathrm{H} 4$ & Neat & Dako TRS & MTM Labs, Germany \\
\hline p63 & $4 \mathrm{~A} 4$ & $1: 20$ & Dako TRS & Chemicon, Temecula, CA \\
\hline SMA & $1 \mathrm{~A} 4$ & $1: 800$ & Dako TRS & Dako \\
\hline SMMS & SMMS-1 & 1:1600 & Dako TRS & Biogenex, San Ramon, CA \\
\hline S100 & Rabbit polyclonal & 1:1600 & Dako TRS & Dako \\
\hline
\end{tabular}


Table 2 Sebaceous and non-sebaceous lymphadenomas

\begin{tabular}{lccc}
\hline Lymphadenomas & Age-Median (range) & Sex (ratio) & Size-Median (range) \\
\hline Sebaceous $(n=22)$ & 63.5 years $(35-84)$ & $9 \mathrm{~F}: 12 \mathrm{M} \mathrm{(1:1.3)}$ & $2.9 \mathrm{~cm} \mathrm{(1-6)}$ \\
Non-sebaceous $(n=11)$ & 65.5 years $(11-78)$ & $8 \mathrm{~F}: 2 \mathrm{M} \mathrm{(4:1)}$ & $1.5 \mathrm{~cm}(0.6-5)$ \\
All lymphadenoma $(n=33)$ & 65 years $(11-84)$ & $17 \mathrm{~F}: 14 \mathrm{M} \mathrm{(1.2:1)}$ & $2.2 \mathrm{~cm}(0.6-6)$
\end{tabular}

Abbreviations: F, female; M, male.

Table 3 Sebaceous lymphadenomas: clinical information

\begin{tabular}{|c|c|c|c|c|}
\hline Cases & Age/sex & Site & Presentation & Size $(\mathrm{cm})$ \\
\hline SL1 & $42 \mathrm{~F}$ & L parotid & Mass & 2.2 \\
\hline SL2 & $48 \mathrm{M}$ & $\mathrm{R}$ parotid & Mass for 6 years & 3.5 \\
\hline SL3 & $67 \mathrm{~F}$ & Parotid & Unknown & 2 \\
\hline SL4 & $84 \mathrm{~F}$ & L parotid & Mass-slowly enlarging & 3.5 \\
\hline SL5 & $74 \mathrm{~F}$ & $\mathrm{R}$ parotid & Mass $^{a}$ & 1.5 \\
\hline SL6 & $74 \mathrm{M}$ & $\mathrm{L}$ parotid & Mass-slowly enlarging & 6 \\
\hline SL7 & $76 \mathrm{M}$ & $\mathrm{R}$ parotid & Mass $^{\mathrm{b}}$ & 5.5 \\
\hline SL8 & $53 \mathrm{~F}$ & $\mathrm{~L}$ parotid & Mass & 2.9 \\
\hline SL9 & $59 \mathrm{~F}$ & $\mathrm{R}$ parotid & Mass & 1 \\
\hline SL10 & $74 \mathrm{M}$ & $\mathrm{R}$ parotid & Mass for 6 months & 2.3 \\
\hline SL11 & $55 \mathrm{M}$ & L neck & Mass & 2.1 \\
\hline SL12 & $53 \mathrm{M}$ & $\mathrm{R}$ parotid & Mass & 5.1 \\
\hline SL13 & $74 \mathrm{M}$ & L parotid & Mass & 3.2 \\
\hline SL14 & $60 \mathrm{M}$ & L parotid & Mass & 1.8 \\
\hline SL15 & $43 \mathrm{~F}$ & $\mathrm{R}$ parotid & Mass & 2.3 \\
\hline SL16 & $76 \mathrm{M}$ & $\mathrm{R}$ parotid & Mass for 2 months & 1.2 \\
\hline SL17 & $35 \mathrm{M}$ & $\mathrm{L}$ parotid & Mass-long standing & 3.1 \\
\hline SL18a & $54 \mathrm{M}$ & $\mathrm{R}$ buccal & Pedunculated mass & Unknown \\
\hline SL18b & Same patient as above & L buccal & Pedunculated mass & Unknown \\
\hline SL19 & $64 \mathrm{~F}$ & $\mathrm{R}$ parotid & Mass for 3 months & 2.2 \\
\hline SL20 & $79 \mathrm{M}$ & $\mathrm{R}$ parotid & Mass for 2 months & 3.5 \\
\hline SL21 & $77 \mathrm{~F}$ & $\mathrm{R}$ parotid & Mass for many years & 3.3 \\
\hline
\end{tabular}

Abbreviations: F, female; L, left; M, male; R, right; SL, sebaceous lymphadenoma.

Duration of presentation, side and location, when known is mentioned.

${ }^{\mathrm{a}}$ Malignant transformation to basal cell.

${ }^{\mathrm{b}}$ Malignant transformation to sebaceous adenocarcinoma.

${ }^{\mathrm{C}}$ Associated with Warthin tumor and sclerosing polycystic adenosis.

Table 4 Non-sebaceous lymphadenomas: clinical information

\begin{tabular}{|c|c|c|c|c|}
\hline Cases & Age/sex & Site & Presentation & Size $(\mathrm{cm})$ \\
\hline NSL1 & $62 \mathrm{~F}$ & L submandibular & Mass & 5 \\
\hline NSL2 & $52 \mathrm{~F}$ & Neck & Mass & 1.1 \\
\hline NSL3 & $76 \mathrm{M}$ & $\mathrm{R}$ parotid & Mass & 1.5 \\
\hline NSL4 $^{\mathrm{a}}$ & $78 \mathrm{~F}$ & $\mathrm{R}$ cervical lymph node & Mass & 0.9 \\
\hline NSL5 & $66 \mathrm{~F}$ & $\mathrm{~L}$ parotid & Mass & 2 \\
\hline NSL6 & $70 \mathrm{~F}$ & $\mathrm{R}$ parotid & Mass & 2.1 \\
\hline NSL7 & $11 \mathrm{~F}$ & $\mathrm{~L}$ parotid & Mass & 3.5 \\
\hline NSL8ab & $70 \mathrm{M}$ & R Intraparotid lymph node & Unknown & 1.2 \\
\hline $\mathrm{NSL8b}^{\mathrm{b}}$ & Same patient as above & Same as above & Unknown & 0.6 \\
\hline NSL9 & $65 \mathrm{~F}$ & L parotid & Mass for 4 months & 1.5 \\
\hline NSL11 & $60 \mathrm{~F}$ & R submandibular & Mass & 2.9 \\
\hline
\end{tabular}

Abbreviations: F, female; L, left; M, male; R, right; NSL, non-sebaceous lymphadenoma.

Duration of presentation when known is mentioned.

${ }^{\mathrm{a}}$ History of low-grade salivary duct carcinoma.

${ }^{\mathrm{b}}$ Associated with chronic lymphocytic leukemia.

for several years including one with breast carcinoma (patients SL16 and SL20; Table 3). Thus, in 7 of the 23 patients $(30 \%)$ there was history of immunomodulatory drug therapy for autoimmune diseases, cancer chemotherapy or preexisting low-grade malignancies. The tumors were resected in 22 of 23 patients, the excisions designated as parotidectomy in 18 (superficial 5, deep 1, and not specified 


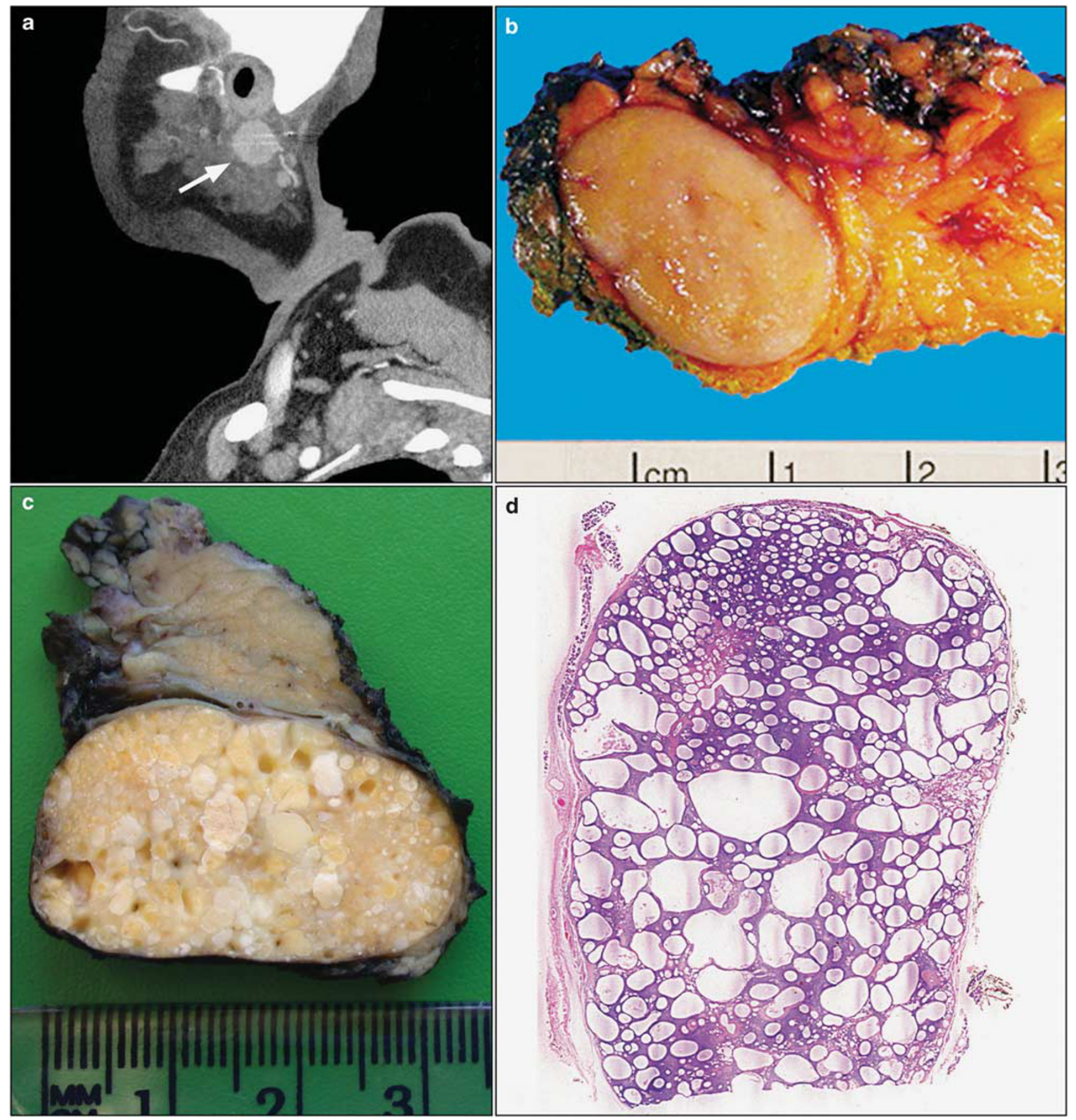

Figure 1 Sebaceous lymphadenoma. (a, b) Sixty-four-year-old woman (SL19): contrast enhanced computerized tomography shows a well-defined mass (arrow) in the right preauricular area (a). The tumor was firm and well circumscribed, measured $2.2 \mathrm{~cm}$ in size and had a tan, homogeneous cut surface (b). (c, d) Seventy-nine-year-old man with a 3.5-cm encapsulated mass in the right parotid (SL20). The cut surface is tan yellow and multicystic (c) with cysts measuring up to $5 \mathrm{~mm}(\mathbf{d})$.

12). No recurrences were noted in 11 patients with follow-up ranging from 1 to 8 years (median 2.5 years).

\section{Pathology}

All tumors were well circumscribed with a median size of $2.2 \mathrm{~cm}$, and 28 of $33(84 \%)$ tumors were encapsulated (Figures 1b-d). Twenty-four of thirtythree $(72 \%)$ tumors were homogenous, multicystic, gray-tan to yellow. The cyst contents in four grossly cystic tumors were gelatinous, yellow sebum like, and chalky white in one case each, and not specified in the fourth tumor.

Microscopically, in sebaceous LAD the epithelial component comprising solid nests, trabeculae, cords, glands, and tubules of basal, glandular, 
squamous, and sebaceous cells (Figures 2a-c). Most cell clusters had a basal cell layer at the periphery, and solid or cystic centers. Tubuloglandular structures showed two cell layers with an outer basal cell layer and an inner luminal glandular cell layer comprising cuboidal or low columnar cells. Keratinization was frequent in squamous cells. Clusters of sebaceous cells, tubules, and cysts with sebaceous cells in lining epithelium were frequent. Cyst contents were keratinous material, eosinophilic proteinaceous fluid, or sebaceous secretions.

In non-sebaceous LAD, the epithelial component comprising predominantly solid cords and trabeculae of basal cells admixed with low columnar cells around tubuloglandular spaces without any sebaceous differentiation (Figures 3a and b). Squamous differentiation with keratinization was seen less frequently $(2 / 11,18 \%)$.

The lymphocytic infiltrate comprising mixed populations of $\mathrm{B}$ and $\mathrm{T}$ lymphocytes was prominent in all LAD, with germinal centers in 28 of $33(84 \%)$ tumors (Figure 2d). Stromal foreign body type giant cell granulomatous reaction (Figure 2d) was frequently noted in association with ruptured cyst in sebaceous $\operatorname{LAD}(n=11 / 22,50 \%)$, and less frequently in non-sebaceous LAD $(n=2 / 11,18 \%)$. Intraepithelial $\mathrm{B}$ and $\mathrm{T}$ lymphocytes were noted in 13 of 13 tumors, but were remarkable in only 1 case (Figure 3b). Five tumors appeared to be intranodal with intact lymph node capsule. Two of these (40\%) also had adjacent normal salivary tissue elements within the lymph node.

Oncocytic change was seen in a combined sebaceous LAD and Warthin tumor that was associated with an adjacent sclerosing polycystic adenosis tumor within the same parotid gland (patient SL17; Table 3).

The epithelial cells were typically negative for mucin by mucicarmine stain ( $8 / 8$ tumors). Intraluminal mucin was occasionally detected (2/8 tumors). Periodic Schiff's stain (with and without diastase) highlighted the basement membrane and stained the intraluminal secretions $(5 / 5)$ cases.

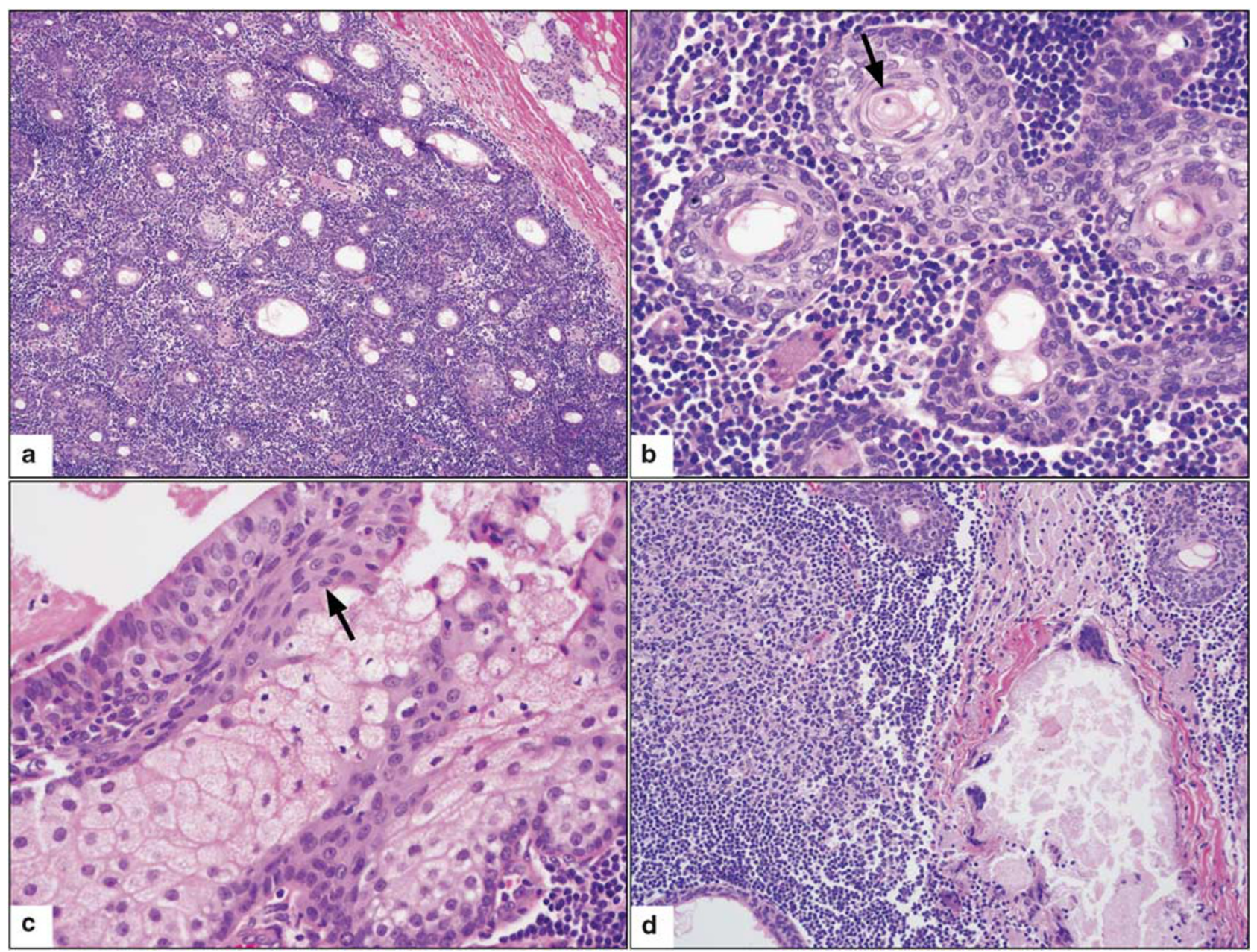

Figure 2 Sebaceous lymphadenoma (a, b from SL19, same case as in Figures 1a and b). The tumor is biphasic with epithelial and lymphoid components, and is separated from the adjacent uninvolved parotid gland by a thin fibrous capsule (a, $\times 10)$. Solid and microcystic clusters with squamous and sebaceous differentiation on the inside and basal cells on the outside. Focal keratinization (arrow) is seen $(\mathbf{b}, \times 40)$. Sebaceous lymphadenoma (SL17) showing a cyst lined by columnar cells, large foamy sebaceous cells, a small area of squamous cells (arrow) and basal cells at the periphery $(\mathbf{c}, \times 40)$. Foreign body type giant cell reaction to ruptured cyst contents is seen adjacent to a lymphoid follicle with germinal center $(\mathbf{d}, \times 20)$. 


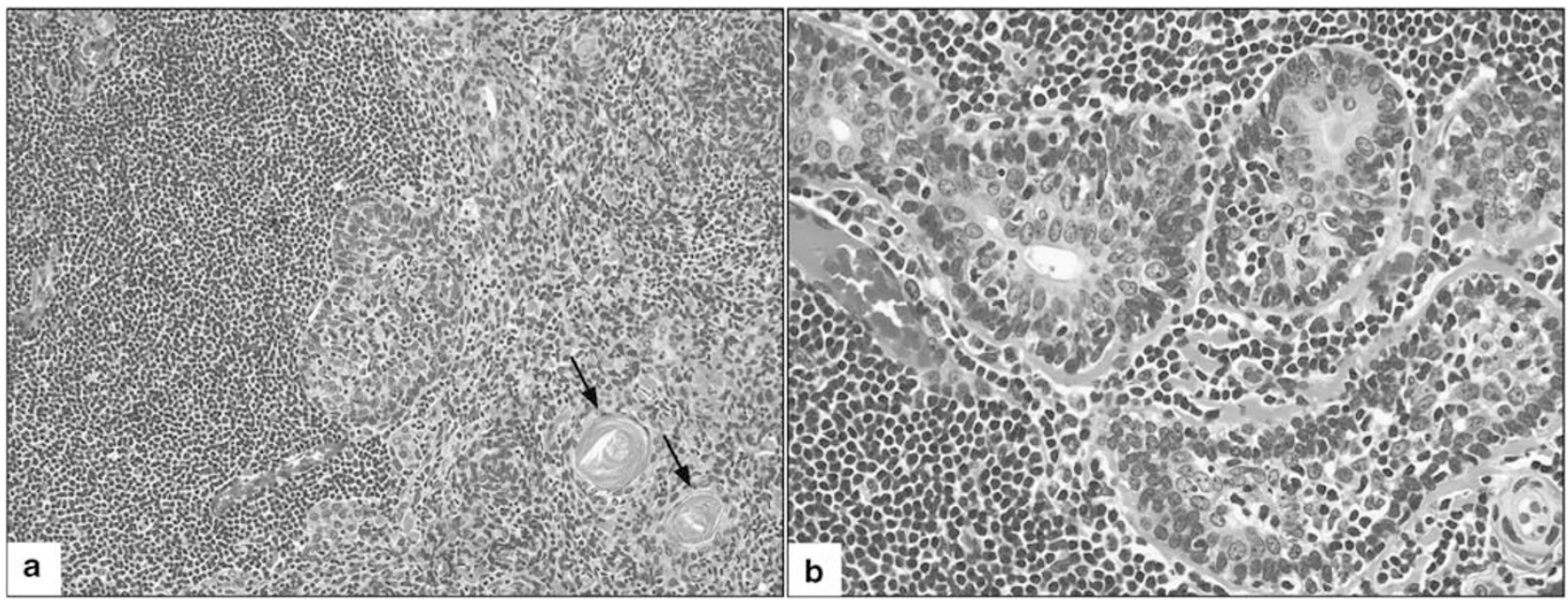

Figure 3 Non-sebaceous lymphadenoma in an 11-year-old girl (NSL7) showing solid clusters of basaloid cells with keratinizing (arrows) squamous cells $(\mathbf{a}, \times 20)$ and low columnar cells lining tubules with intraepithelial lymphocytes $(\mathbf{b}, \times 40)$.

Table 5 Ancillary studies in lymphadenomas

\begin{tabular}{|c|c|c|c|}
\hline Study & Sebaceous lymphadenoma & Non-sebaceous lymphadenoma & All tumors \\
\hline \multicolumn{4}{|l|}{ Immunohistochemistry } \\
\hline \multicolumn{4}{|l|}{ Basal cell markers } \\
\hline p63 & $11 / 11$ & $7 / 7$ & 18/18 (100\%) \\
\hline HMWK & $3 / 3$ & $2 / 2$ & $5 / 5(100 \%)$ \\
\hline CK5/6 & $8 / 8$ & $1 / 1$ & $9 / 9(100 \%)$ \\
\hline \multicolumn{4}{|l|}{ Epithelial markers } \\
\hline CK7 & $8 / 8$ & $4 / 4$ & $12 / 12(100 \%)$ \\
\hline CK20 & $1 / 7$ & $0 / 4$ & $1 / 11(0.9 \%)$ \\
\hline $\begin{array}{l}\text { Myoepithelial markers (calponin, } \\
\text { SMA, SMMS, S100) }\end{array}$ & $3 / 8$ & $3 / 8$ & $6 / 16(37.5 \%)$ \\
\hline Proliferation index (Ki-67) & $0 / 3$ & $0 / 2$ & $0 / 5$ \\
\hline \multicolumn{4}{|l|}{ Lymphoid component } \\
\hline Reactive mixed $\mathrm{B}$ and $\mathrm{T}$ cells & $4 / 4$ & $2 / 4^{\mathrm{b}}$ & $6 / 8^{\mathrm{b}}(75 \%)$ \\
\hline \multicolumn{4}{|l|}{ Viral markers } \\
\hline HPV & $0 / 9$ & $0 / 2$ & $0 / 11(0 \%)$ \\
\hline P16 & $7 / 8$ & $0 / 2$ & $0 / 10^{\mathrm{c}}$ \\
\hline HPV-ISH & $0 / 5$ & $0 / 2$ & $0 / 7$ \\
\hline EBV & $0 / 7$ & $0 / 6$ & $0 / 13(0 \%)$ \\
\hline EBER & $0 / 7$ & $0 / 4$ & $0 / 11$ \\
\hline EBV-LMP & - & $0 / 3$ & $0 / 3$ \\
\hline HHV-8 (KSHV) & $0 / 6$ & $0 / 2$ & $0 / 8(0 \%)$ \\
\hline
\end{tabular}

${ }^{\mathrm{a}}$ Proliferation index was evaluated in epithelial cells and considered negative when $<5 \%$.

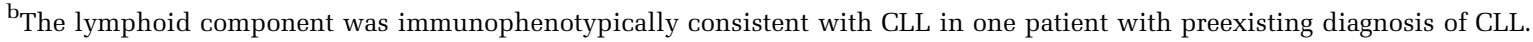

${ }^{\mathrm{C}}$ Strong, diffuse nuclear and cytoplasmic expression of p16 has been correlated with HPV infection and was not seen in any tumor.

IHC results are summarized in Table 5 . The basal cells were positive for at least one of the basal cell markers (p63, CK5/6, and/or high molecular weight keratin) in all cases that were tested. In tubuloglandular structures, the luminal cells expressed CK7 while the abluminal basal cells, sebaceous and squamous cells were negative. All tumors were negative for CK20 except for focal expression in a sebaceous LAD. Myoepithelial markers (calponin, smooth muscle actin, smooth muscle myosin, and/ or S100 protein) were negative in 10 of 16 cases $(63 \%)$, and focally expressed in a few abluminal basaloid cells in 5 tumors. Proliferation marker (Ki-67) showed appropriately intense expression in the germinal centers but absent or occasional expression $(<5 \%)$ in epithelial cells. The lymphoid population was reactive and mixed B- and T-cell types. The germinal centers were populated by B cells (CD20 and/or CD79a positive) and the interfollicular areas by T cells (CD3 and/or CD5) as seen in reactive follicular hyperplasia. Intraepithelial lymphocytes were T-cell type in one, and mixed B-and T-cell types in another tumor where their immunophenotype was examined. In one patient 
with two non-sebaceous LAD in the right parotid, the lymphoid infiltrate was malignant, positive for CD20, CD5, CD23, and negative for cyclin D1, consistent with his preexisting chronic lymphocytic leukemia (patient NSL8a,b in Table 4; Figures 4a-c).

\section{Viral Studies}

Focal weak-to-moderate p16 expression was noted in epithelial cells in 8 of 10 cases, but none showed strong, diffuse nuclear and cytoplasmic expression of p16 as is typical of HPV-associated squamous cell carcinomas. None of the tumors tested were positive for HPV, EBV, and HHV-8 (Table 5).

\section{Malignant Transformation and Associated Salivary Gland Pathology}

Two sebaceous LADs showed malignant transformation: a 74-year-old woman developed basal cell adenocarcinoma (patient SL5; Table 3; Figures 5a and b) and a 76-year-old man developed sebaceous carcinoma (patient SL7; Table 3; Figures 5c and d). Both tumors showed benign component and areas of transition from benign to malignant (Figures $5 \mathrm{~b}$ and c). The diagnosis of malignancy was based on mitoses and increased cellularity in basal cell adenocarcinoma where the tumor cells remained monomorphic (Figure 5a). In the sebaceous carcinoma, there was significant cytologic atypia, nuclei were large and vesicular with prominent nucleoli, and the cytoplasm was foamy (Figures 5c and d). A 70-year-old man with preexisting chronic lymphocytic leukemia developed two non-sebaceous LAD in two intraparotid lymph nodes in the right parotid (patient NSL8; Table 4; Figure 4). A 78-year-old woman with intranodal nonsebaceous LAD in the right neck had prior history of low-grade intraductal carcinoma in the right parotid gland (patient NSL4; Table 4). The LAD was morphologically not consistent with metastatic adenocarcinoma. No follow-up was available in these patients after resection of their LAD. A 35-year-old man (patient SL17; Table 3) had combined sebaceous LAD and Warthin tumor associated with an adjacent but separate sclerosing polycystic adenosis tumor.
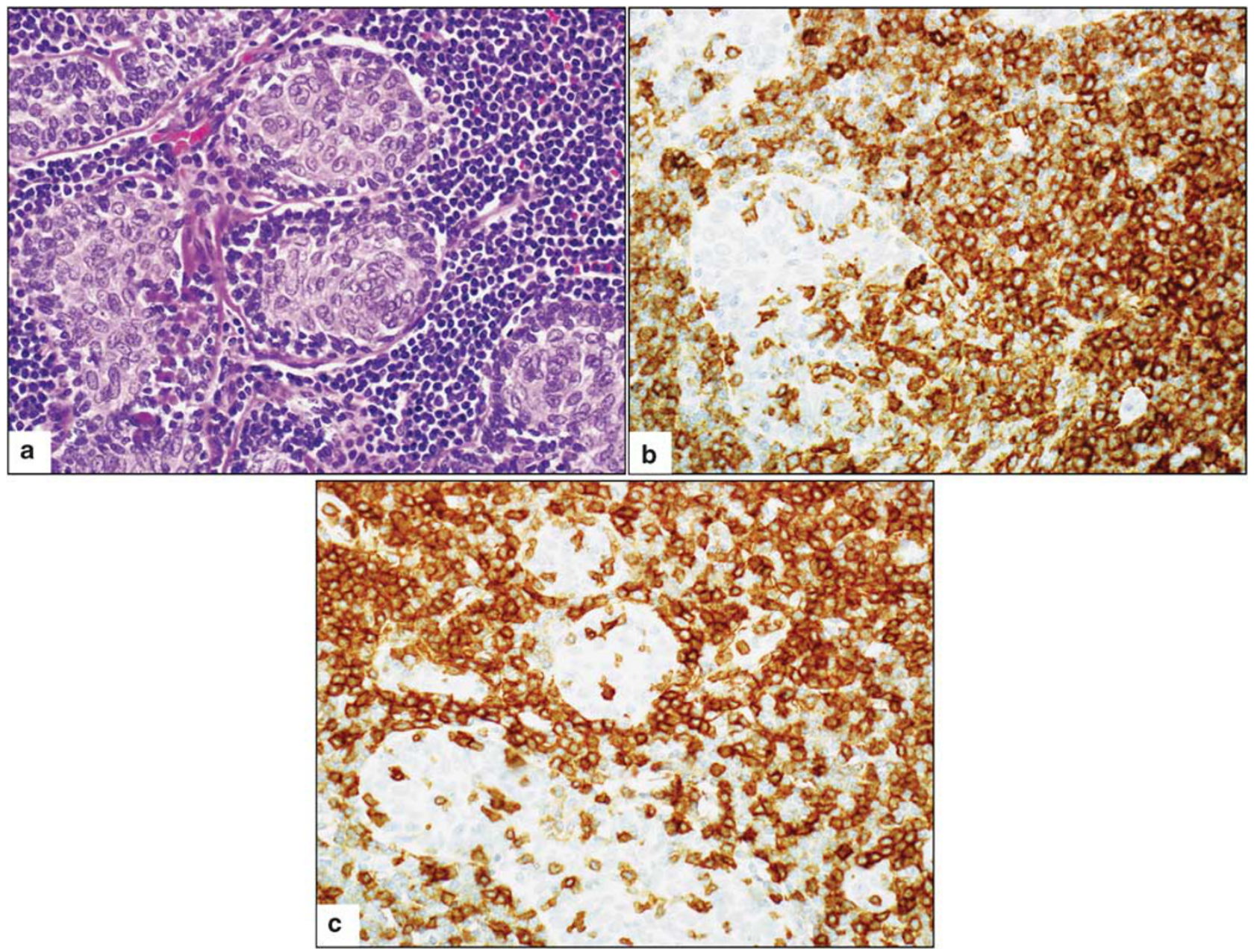

Figure 4 (a-c) Non-sebaceous lymphadenoma in a 70-year-old man with chronic lymphocytic leukemia (NSL8). The basaloid epithelial cells are arranged in solid clusters and cords with intraepithelial and stromal malignant small B lymphocytes $(\mathbf{a}, \times 40)$ that are positive for CD20 (b, $\times 40)$ and CD5 $(\mathbf{c}, \times 40)$. 


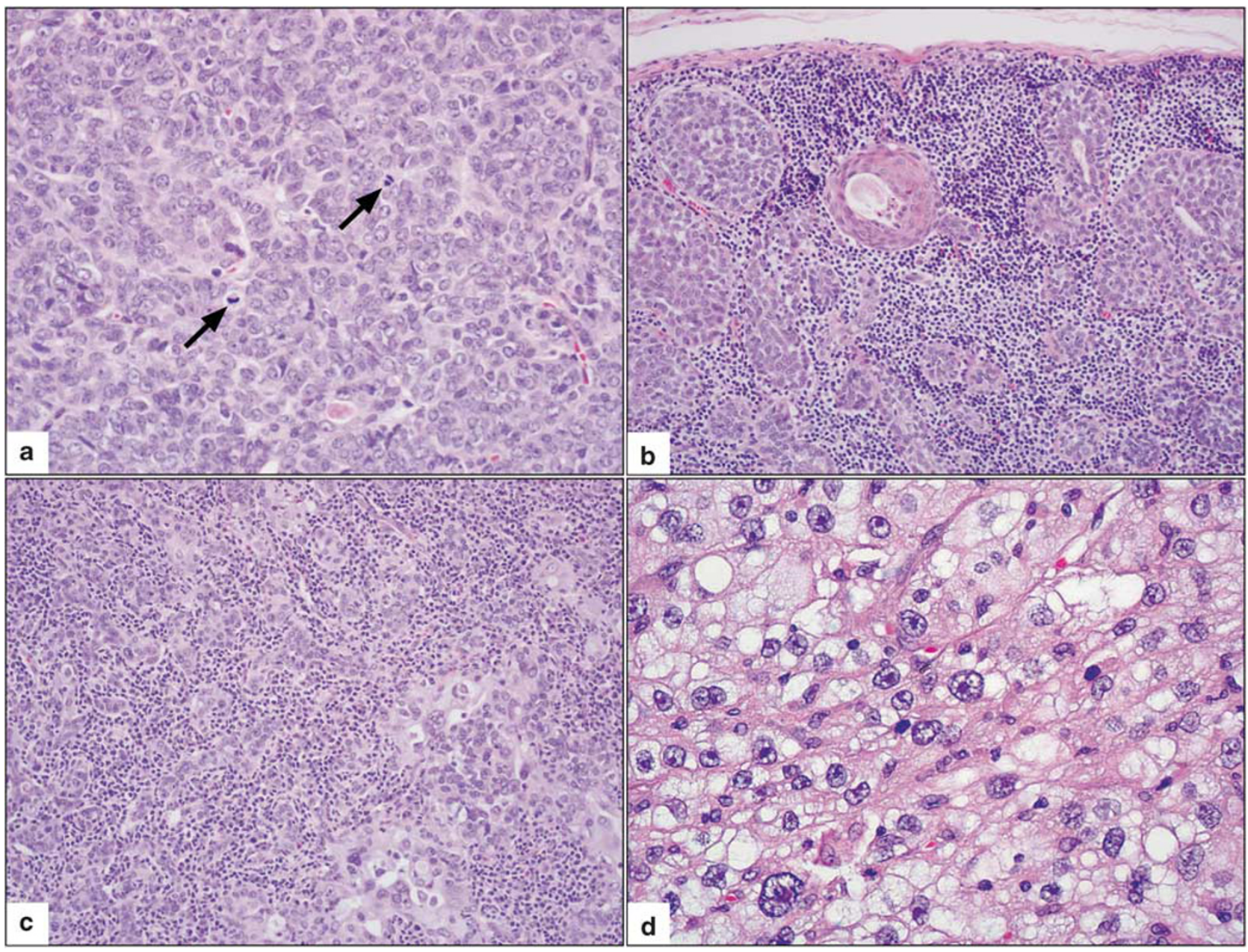

Figure 5 Malignant transformation in lymphadenoma. (a-b) Seventy-four-year-old man with $1.5 \mathrm{~cm}$ sebaceous lymphadenoma in the right parotid gland. Tumor shows an area of basal cell adenocarcinoma comprising sheets of monomorphic basaloid cells with frequent mitoses (arrows, $\mathbf{a}, \times 40$ ). The capsule is intact and benign areas consistent with lymphadenoma are present within the tumor $(\mathbf{b}, \times 40)$. (c, d) Sebaceous carcinoma arising in a sebaceous lymphadenoma with area of transition between benign (upper left) and malignant (lower right) epithelial cells $(\mathbf{c}, \times 40)$. Tumor cells show significant cytologic atypia with abundant foamy cytoplasm, large pleomorphic vesicular nuclei, and prominent nucleoli $(\mathbf{d}, \times 40)$.

\section{Discussion}

We present clinicopathological and immunophenotypic profile of 33 salivary LAD, the largest series to date. Our observations confirm that LADs are typically benign tumors affecting adults over the age of 50 years $(84 \%)$, and two thirds of them show some sebaceous differentiation. Interestingly, $48 \%$ of patients in the current series suffered from additional co-morbidities, including a subset $(30 \%)$ that received immunomodulatory drug therapy for autoimmune diseases and/or chemotherapy for cancers, or had associated low-grade malignancies, raising the question of altered immune status as a predisposing factor. Multiple co-morbidities may be coincidental given the advanced age of most patients, although chronic diseases may further alter immune status.

We questioned a possible viral etiology but found no evidence of EBV, HPV, and HHV-8 viruses.
Negative results with in situ hybridization for EBER have been reported previously in three non-sebaceous LAD. ${ }^{6,16}$ We add another 13 tumors that were negative for EBV. To the best of our knowledge, this is the first study exploring the role of HPV or HHV-8 viruses in LAD.

Sebaceous LAD showed a slight male preponderance (1.3-1). This is similar to other sebaceous salivary gland tumors. ${ }^{2}$ This may be due to the generally higher concentration of sebaceous glands in men than in women, associated with the higher density of facial hair in the former. Additionally, the site distribution of sebaceous LAD mirrors the distribution of sebaceous rests seen in salivary glands and the oral cavity. Sebaceous cells have been found in 30-40\% of normal adult parotid glands ${ }^{3}$ and $6 \%$ of submandibular glands. ${ }^{2}$ Fordyce granules in the buccal mucosa comprising sebaceous glands and may be seen in as many as $80 \%$ of normal adults. ${ }^{3}$ Despite this, salivary tumors with 
sebaceous differentiation are rare. Intraoral sebaceous LAD was first reported by Gnepp and Brannon, ${ }^{2}$ included in a series of 21 sebaceous tumors of the salivary gland. Origin in the Fordyce granuloma has been proposed. ${ }^{19}$

To date, 11 non-sebaceous LAD have been reported in the literature, the largest series comprising three cases only. ${ }^{6,16}$ We add another 11 cases. Unlike their sebaceous counterparts, the non-sebaceous tumors were more common in women than in men, similar to most salivary gland tumors. Additionally, $40 \%$ of the patients had extraparotid nonsebaceous LAD in contrast to sebaceous LAD that was intraparotid in nearly $90 \%$ of patients. No other demographic, clinical, or pathologic differences were found in these two subtypes. Some investigators argue that non-sebaceous LAD may not be a distinct entity but rather a basal cell adenoma or cystadenoma with extensive lymphoid infiltrate. $^{4,6,9,11}$ The non-sebaceous LAD tend to have more focal keratinization, less peripheral palisading, generally lack the production of basement membrane material and have a small or non-existent myoepithelial component as compared with basal cell adenomas. ${ }^{6,12-14,16}$

The prominent reactive mixed $\mathrm{B}$ - and T-cell proliferation with germinal centers is identical to follicular hyperplasia of reactive lymph nodes, and is a defining feature of LAD. We frequently noted a granulomatous reaction in sebaceous LAD, likely secondary to ruptured cyst contents. The possibility that LAD are intranodal tumors arising in intranodal sebaceous or salivary inclusions akin to Warthin tumor has been proposed. ${ }^{1-3}$ In a recent report, Dardick and Thomas ${ }^{13}$ used a definition of nonsebaceous LAD that excluded cases with any evidence of lymph node architecture. Our diagnostic criteria are admittedly more liberal in that one sebaceous and four non-sebaceous LADs in the current series were intranodal, three in cervical lymph nodes, and two in intraparotid lymph nodes. Intraparotid and cervical nodes in the vicinity of the parotid may have salivary inclusions, and as such may develop primary intranodal salivary gland neoplasms, both benign and malignant. Thus, we felt that it was justifiable to retain these cases for this study. In fact, two of the intranodal tumors also had normal salivary tissue elements within the lymph node.

LADs are exceedingly rare in children with only four reported cases (Table 6)..$^{6,16,20,21}$ To our knowledge, the 11-year-old girl in the present series is the youngest patient with LAD. Table 6 shows a trend to non-sebaceous LAD in the children. This may be because sebaceous differentiation in salivary glands develops later in life and is not present in infants and children. ${ }^{3}$

Mayorga et $a^{22}$ reported a sebaceous LAD arising in association with acinic cell carcinoma in the parotid gland in a 78-year-old woman. The current series includes seven patients with synchronous or
Table 6 Review of lymphadenomas in children

\begin{tabular}{|c|c|c|c|c|}
\hline Reports & $\begin{array}{l}\text { Age/ } \\
\text { sex }\end{array}$ & Lymphadenoma & Location & $\begin{array}{l}\text { Size } \\
(\mathrm{cm})\end{array}$ \\
\hline Ma et $a l^{6}$ & $13 \mathrm{M}$ & Non-sebaceous & Preauricular & 3 \\
\hline Rawlinson et $a l^{20}$ & $13 \mathrm{~F}$ & Sebaceous & Left parotid & 2.4 \\
\hline Chang et $a l^{16}$ & $15 \mathrm{~F}$ & Non-sebaceous & Right parotid & 4.5 \\
\hline Sun et $a l^{21}$ & $16 \mathrm{M}$ & Sebaceous & Left parotid & 2.4 \\
\hline Current series & $11 \mathrm{~F}$ & Non-sebaceous & Left parotid & 3.5 \\
\hline
\end{tabular}

metachronous salivary or non-salivary malignant tumors. Two sebaceous LAD in the current series showed transformation into basal cell adenocarcinoma and sebaceous carcinoma, respectively. Carcinomatous change is rare in LAD, and should be suspected when the epithelial component in an otherwise typical LAD begins to show frequent mitoses and significant cytologic atypia with areas of transition from benign to malignant, even in the absence of infiltration of the tumor capsule. Among the five reported cases of sebaceous lymphadenocarcinoma including one in the current series and four in the literature, there were four men and one woman. ${ }^{2,23,24}$ Rarely, LAD may be associated with other benign salivary tumors as in one of our cases where a 35-year-old man had a combined sebaceous LAD and Warthin tumor, adjacent to a separate sclerosing polycystic adenosis tumor. ${ }^{18}$

Both sebaceous and non-sebaceous LAD raise several differential diagnostic considerations, ${ }^{13}$ most important being metastatic low-grade adenocarcinoma and adenosquamous carcinoma involving intraparotid lymph node. Nodal metastasis is not the primary presentation in low-grade adenocarcinomas. Mitoses in LAD are restricted to the germinal centers in the lymphoid component, and were absent or rare in the epithelial cells. Epithelial cells in LAD do not show cellular atypia or other overt features of malignancy. The lack of sinusoidal pattern of epithelial growth may be helpful in ruling out nodal metastasis. Primary or metastatic lymphoepithelial carcinoma may mimic non-sebaceous LAD; however, severe atypia, brisk mitosis, infiltrating growth, and frequent association with EBV distinguish them from benign tumors. Tumor cell nests in lymphoepithelial carcinoma tend to be more syncytial in nature, comprising cells with indistinct membranes and characteristic large vesicular nuclei with prominent nucleoli. Mucoepidermoid carcinoma may occasionally show prominent lymphoid stroma and focal sebaceous differentiation. Identification of the three basic cell types, epidermoid, intermediate and, especially, mucous cells, and the absence of keratinization are useful features in recognizing mucoepidermoid carcinoma. Additionally, when present, infiltration may help distinguish mucoepidermoid carcinoma from LAD. Occasionally, the lymphoid component may lead to the consideration of lymphoma. Demonstration of 
mixed $\mathrm{B}$ and $\mathrm{T}$ cells and lack of light chain restriction should help rule it out. Among benign conditions lymphoepithelial cysts, lymphoepithelial sialadenitis and other salivary gland tumors with sebaceous differentiation may need to be distinguished from LAD. Lymphoepithelial cysts tend to be multifocal and bilateral, and do not have the distinct circumscription and encapsulation seen in LAD. Lymphoepithelial sialadenitis tends to involve the gland diffusely. Of note, none of our LAD arose in the setting of lymphoepithelial sialadenitis. In fine needle aspirations, LAD may be mistaken for Warthin tumor due to their lymphoid contents, ${ }^{15,21}$ but the absence of oncocytic cells should help rule out the latter.

In summary, LADs are encapsulated, benign solid and/or cystic tumors of major salivary glands affecting patient in their sixth to eighth decade. A remarkable female preponderance was noted for non-sebaceous LAD, similar to other salivary gland tumors, and in contrast to sebaceous LAD, that showed a slight male predilection. The former is also more frequently extraparotid compared with the latter. The prominent reactive lymphoid proliferation is a defining feature. Basal cells appear to be the most predominant epithelial component with frequent sebaceous and squamous differentiation, and show little proliferative activity. Myoepithelial cell participation appears to be insignificant in this neoplastic process. Synchronous or metachronous salivary or non-salivary tumors may be associated with some LAD. Malignant transformation may rarely occur in the epithelial component. Although the etiology remains unknown, altered immune status may be a risk factor in a subset of patients.

\section{Disclosure/conflict of interest}

The authors declare no conflict of interest.

\section{References}

1 McGavran MH, Bauer WC, Ackerman LV. Sebaceous lymphadenoma of the parotid salivary gland. Cancer 1960;13:1185-1187.

2 Gnepp DR, Brannon R. Sebaceous neoplasms of salivary gland origin. Report of 21 cases. Cancer 1984;53:2155-2170.

3 Batsakis JG, el-Naggar AK. Sebaceous lesions of salivary glands and oral cavity. Ann Otol Rhinol Laryngol 1990;99:416-418.

4 Auclair PL. Tumor-associated lymphoid proliferation in the parotid gland. A potential diagnostic pitfall. Oral Surg Oral Med Oral Pathol 1994;77:19-26.

5 Bos I, Meyer S, Merz H. Lymphadenoma of the parotid gland without sebaceous differentiation. Immunohistochemical investigations. Pathologe 2004;25:73-78.
6 Ma J, Chan JK, Chow CW, et al. Lymphadenoma: a report of three cases of an uncommon salivary gland neoplasm. Histopathology 2002;41:342-350.

7 Gnepp DR, Henley JD, Simpson RHW, et al. Salivary and Lacrimal glands. In: Gnepp DR (ed). Diagnostic Surgical Pathology of the Head and Neck. 2nd edn. Elsevier: Philadelphia, 2009, pp 413-562.

8 Kwon GY, Kim EJ, Go JH. Lymphadenoma arising in the parotid gland: a case report. Yonsei Med J 2002;43:536-538.

9 Chang JY, Hsiao CH. Lymphadenoma lacking sebaceous differentiation in the parotid gland. J Formos Med Assoc 2004;103:459-462.

10 Musthyala NB, Low SE, Seneviratne RH. Lymphadenoma of the salivary gland: a rare tumour. J Clin Pathol 2004;57:1007.

11 Cheuk W, Chan JK. Advances in salivary gland pathology. Histopathology 2007;51:1-20.

12 Yang S, Chen X, Wang L, et al. Non-sebaceous lymphadenoma of the salivary gland: case report with immunohistochemical investigation. Virchows Arch 2007;450:595-599.

13 Dardick I, Thomas MJ. Lymphadenoma of parotid gland: Two additional cases and a literature review. Oral Surg Oral Med Oral Pathol Oral Radiol Endod 2008;105:491-494.

14 Gallego L, Junquera L, Fresno MF. Non-sebaceous lymphadenoma of the parotid gland: immunohistochemical study and DNA ploidy analysis. Oral Surg Oral Med Oral Pathol Oral Radiol Endod 2009;107:555-558.

15 Castelino-Prabhu S, Li QK, Ali SZ. Nonsebaceous lymphadenoma of the parotid gland: cytopathologic findings and differential diagnosis. Diagn Cytopathol 2010;38:137-140.

16 Chang KT, Chadha NK, Leung R, et al. Lymphadenoma: case report of a rare salivary gland tumor in childhood. Pediatr Dev Pathol 2010;13:331-337.

17 Begum S, Gillison ML, Ansari-Lari MA, et al. Detection of human papillomavirus in cervical lymph nodes: a highly effective strategy for localizing site of tumor origin. Clin Cancer Res 2003;9:6469-6475.

18 Gnepp DR, Wang LJ, Brandwein-Gensler M, et al. Sclerosing polycystic adenosis of the salivary gland: a report of 16 cases. Am J Surg Pathol 2006;30:154-164.

19 Abdool RA, Solomon LW, Papageorge MB. A clinicopathologic correlation. Sebaceous lymphadenoma. J Mass Dent Soc 2008;57:36-37.

20 Rawlinson NJ, Almarzooqi S, Nicol K. Sebaceous lymphadenoma of the parotid gland in a 13-year-old girl: a case report. Head Neck Pathol 2010;4:144-147.

21 Sun G, Hu Q, Huang X, et al. Sebaceous lymphadenoma of parotid gland in a child. Oral Surg Oral Med Oral Pathol Oral Radiol Endod 2009;107:253-255.

22 Mayorga M, Fernandez N, Val-Bernal JF. Synchronous ipsilateral sebaceous lymphadenoma and acinic cell adenocarcinoma of the parotid gland. Oral Surg Oral Med Oral Pathol Oral Radiol Endod 1999;88:593-596.

23 Linhartova A. Sebaceous glands in salivary gland tissue. Arch Pathol 1974;98:320-324.

24 Croitoru CM, Mooney JE, Luna MA. Sebaceous lymphadenocarcinoma of salivary glands. Ann Diagn Pathol 2003;7:236-239. 